\title{
A Fast and Efficient Dehydration Process for Waste Drilling Slurry
}

\author{
Guo Zheng ${ }^{1, a}$, Cailin Wang ${ }^{1}$ and Yu Sun ${ }^{1}$ \\ ${ }^{1}$ School of Environment and Chemical Engineering, Tianjin Polytechnic University, Tianjin 300387, China
}

\begin{abstract}
In this article, slurry system was converted to colloid from fluid with the colloidization of high polymer coagulants with high viscosity. The solid-liquid separation of the waste slurry was realized by the process of chemical colloidal gel breaking, coagulation function, acidification gelout. In addition, the surface morphology of slurry cake was investigated by using Field emission scanning electron microscope (FE-SEM). The results indicate that mud separation effect is decides on the type of flocculants, gel breaker. The solid content of mud cake increases from $40.5 \%$ to $77.5 \%$ when A-PA and $\mathrm{H} 20$ are employed as the flocculants, gelout, with the dosage of zero point four grams and zero point five grams.
\end{abstract}

\section{Introduction}

Drilling sludge, as a key material in the oil and gas well drilling operation, is used to flush wellhead[12]. However, according to the survey, the annual production of waste drilling sludge is increasing trend year by year in China[3]. Without processing direct emissions, it cause serious environmental problems[4-6]. Because the waste drilling mud contains a large number of organic and inorganic pollutants, without processing direct emissions, it not only takes up a lot of land area, soil compaction and arbitrary term deposits, some harmful substance by rain flushing would leak into the surrounding soil, vegetation, livestock and so on. At present, the chemical solid-liquid separation method is more, through the mud to join the breaker to remove the mud, through mechanical means to achieve solid liquid separation[7]. This article through the electrical repulsion to further strengthen the colloidal properties of the mud, the fluid completely transformed into jelly, using colloidal gel breaking, poly sink properties to achieve a slurry of solid - liquid separation.

\section{Experimental materials and methods}

\subsection{Materials}

Polymer coagulating agent A-PA (anionic), C-PA (cationic), N-PA (non-ionic), industrial pure, Tianjin university of technology textile auxiliaries co, LTD.; H20, industrial pure, Tianjin university of technology textile auxiliaries co, LTD.; sulphuric acid, nitric acid, hydrochloric acid and boric acid, hydrogen peroxide, Tianjin North Asclepius chemical reagent factory; calcium chloride, aluminum

\footnotetext{
${ }^{a}$ Corresponding author: zhengguo0703@126.com.org
} 
sulphate, poly-aluminium chloride, calcium hydroxide, ferric chloride, analysis of pure. Tianjin Kermel Chemical Reagent Co. LTD.

\subsection{Equipment}

Analytical balance, FA2004N, Shanghai Jing marine instrument Co., Ltd.; PHS-25, Shanghai Precision Scientific Instrument Co., Ltd.; circulating water vacuum pump, SHZ-D (III) type, Gongyi City, granting China Instrument Co., Ltd.; precision timing electromotive stirrer, jj-1, Jintan City Liangyou instrument is limited; field emission scanning electron microscopy, Quanta 200, Fei company, chemical oxygen demand (COD) rapid determination instrument, 5B-3A (V7) type and even China Lanzhou Environmental Protection Technology Co., Ltd.; inductively coupled plasma atomic emission spectrometry (ICP-AES), Varian 715-ES, spectro analysis instrument company.

\subsection{Mud pretreatment}

Due to the long time, some performance of the waste drilling sludge is influenced by the gravity. For example, the rheological property becomes weak, the colloid ratio decreases, the precipitation rate increases, and the continuous network structure of the lower sludge is much stronger than that of the continuous net structure of the upper sludge. In order to ensure the consistency of the overall performance of the sludge, mixing must be carried out before use.

\subsection{Slurry solid-liquid separation process}

It takes one hundred take 100 gram of pretreatment of waste drilling mud, adding organic coagulant, mixing, stirring speed was 200 revolutions per minute, until the mud together to form a group and no longer adhere to the wall of the beaker and the treatment of colloidal mud jelly, no liquidity. Then it does to break the glue, which needs to add the gel breaker, shear type stirring, stirring speed of 300350 revolutions per minute, until the existence of no large block of plastic. Coagulant aid: adding coagulant, shear type stirring, stirring speed of R 150-200 revolutions per minute, to small particles and large particles of suspended particles. The vacuum filtration time is $20-30 \mathrm{~min}$, and the vacuum pressure maintained is at $0.08-0.09 \mathrm{MPa}$. All of the above process temperature is 25 degrees centigrade, the relative humidity is $50 \%$.

\subsection{Preparation of mud cake leaching solution and determination of COD and heavy metal ions}

Reference GB5086.2-1997solid waste leaching toxicity level oscillation method and execute GB5085.3-2007hazardous waste identification standard Leaching toxicity identification.

Under natural conditions, the solid state of the waste drilling mud after solid liquid separation is achieved in a static state. Therefore, take 100 gram (dry weight), placed in the 2 litre polyethylene wide mouth bottle (with a sealing plug), adding 1000 milliliter de ionized water, static soak 24 hours, take the supernatant for analysis COD. Finally, the supernatant was filtered through micro porous filter of 0.45 micron, and the filtrate was used for the analysis of heavy metal ions.

\subsection{Field emission microscope}

Take waste drilling mud sample field emission microscope to observe the morphology of the particles. After solid-liquid separation, it takes a small amount of sample to field emission microscope to observe the morphology of the particles. 


\subsection{Technological process}

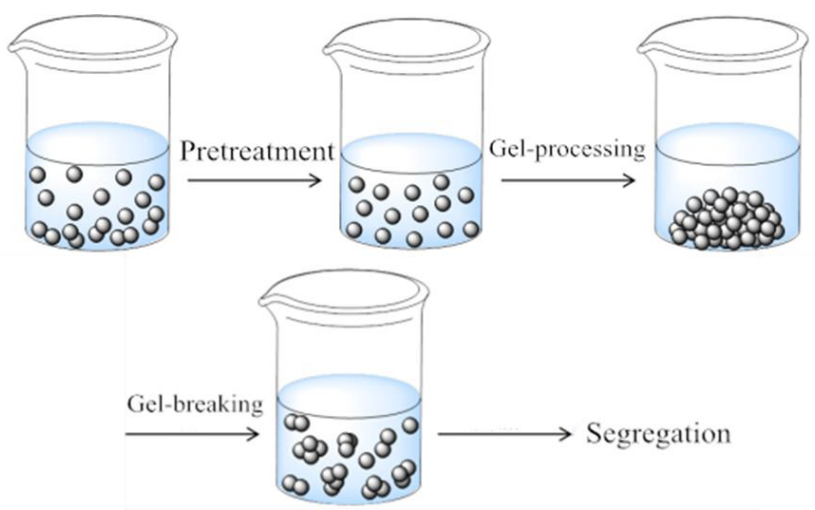

Figure 1. Process flow diagram of waste drilling mud on solid-liquid separation

\section{Results and discussion}

\subsection{Influencing factors of solid-liquid separation of waste drilling mud}

\subsubsection{Effect of type of coagulant on the effect of solid liquid separation}

Respectively we choose three different types of coagulants. They are A-PA, C-PA and N-PA. The separation effect is shown in figure 2.The type of flocculants is a decisive factor for mud solid-liquid separation process; with the increasing of dosage of C-PA, solid content of mud cake is less, which is a negative growth trend. But the using of A-PA significantly enhances the waste mud solid-liquid separation effect. Therefore the changing of N-PA dosing quantity does not affect the basic abandoned drilling mud solid-liquid separation effect. The main reason is that the charge neutralization makes mud dilute, losing colloidal properties. In alkaline condition, A-PA occurs hydrolysis. Some of its amide groups form negative charges, which repel solid phase particles of mud .Because the surface of solid phased particles in the mud is contained negative charge. Repulsive force between the molecules stretch makes molecular structure morphology of polymer flocculants stretch and increases Zeta potential increase of clay particles. The whole system gets into a more stable colloidal system on the apparent. It helps to take advantage of the settlement of colloid to realize solid-liquid separation performance. Therefore, in this solid-liquid separation process of slurry, we choose A-PA flocculants.

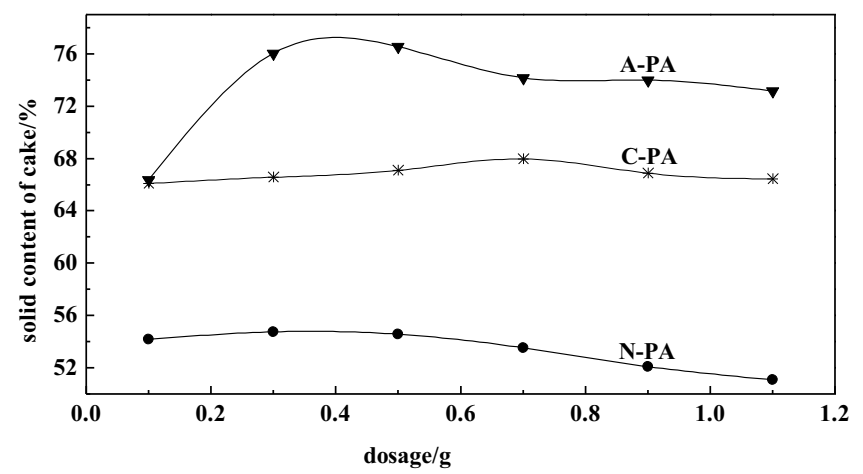

Figure 2. Effect of coagulant type on the separation. 


\subsubsection{Effect of A-PA dosage on the effect of solid liquid separation}

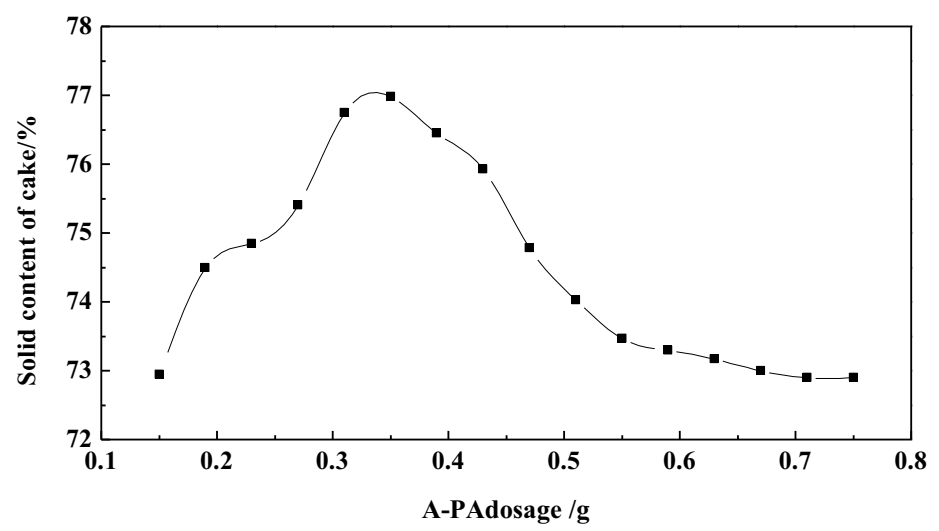

Figure 3. Effect of A-PA dosage on the separation T.

Figure 3 shows the effect of A-PA dosage on the solid liquid separation. With the increasing of A-PA dosage, the solid content of the cake increases first and then decreases. This is mainly because some free water reform to bound water in the mud and cannot realize effective separation. And with the increasing of A-PA, the surface of mud solid particles will adsorb amounts of high molecular material, and then form a layer of protective film in the solid particles, Therefore, in solid-liquid separation process of slurry, the optimal dosage of A-PA is $0.375 \mathrm{~g}$ and the solid content of the mud cake reached the maximum value of $80.5 \%$.

\subsubsection{Effect of gel breaking agent on the effect of solid liquid separation}

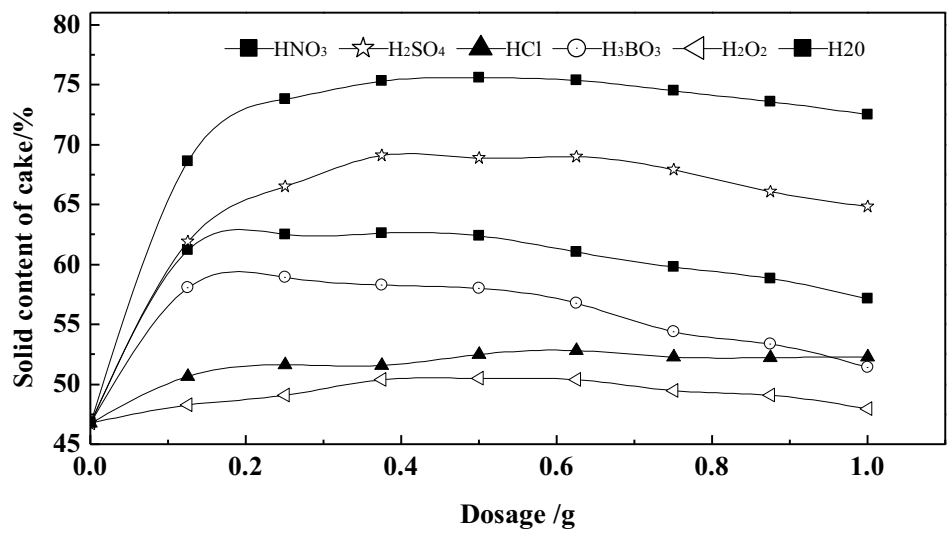

Figure 4. Effect of acidifier dosage on the separation

The effect of adding amount of gel breaker on the solid-liquid separation is shown in Figure 4 . The addition of gel breakers is beneficial to the solid-liquid separation of slurry, but the separation effect is different. Among them, the effect of H20 is best. This main reason is that drilling mud is strong alkaline. And in the alkaline environment, oil and mud treatment agent can combine together and form high stability. By adding acid gel breaker, it can happen neutralization reaction and form complex salt, which changes the balance of the original system and destructs electric double layer. However, nitric acid, sulfuric acid and hydrochloric acid belong to strong acid, which are corrosive, they not only can 
neutralize alkaline and destroy part of waste drilling mud lattice structure of clay particles, reducing the solid-liquid separation effect. Boric acid and hydrogen peroxide are weak acid, which can't completely break the glue. The acid strength of $\mathrm{H} 20$ is in middle position. And the best dosage of H20 is $0.5 \mathrm{~g}$.

\subsection{Micro morphology of waste drilling mud}

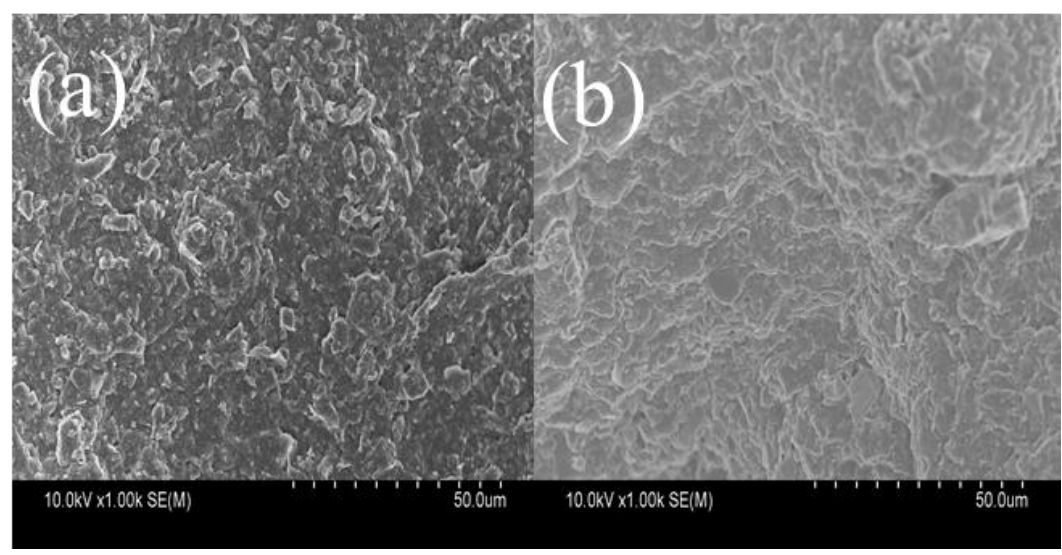

(a) mud cake before the solid-liquid separation (b) mud cake after the solid-liquid separation

Figure 5. SEM micrographs of mud cake

The micro morphology of the waste drilling slurry before and after solid-liquid separation is shown in Figure 5(a,b). Figure 5(a) shows that waste drilling mud contains different particle size of solid particles and oil drops. There are a lot of free water, solid phase particles scattered irregular, no obvious means of gathered phenomenon. The figure 5(b) shows that the mud almost contains litter free water in the mud cake after solid-liquid separation. And solid-phase material arranges closely and gathers big particles. Because the waste drilling mud treatment of moisture content is about $55.5 \%$, all kinds of solid particles, oil drops and water are unable to form a uniform system, a mixed system of water in oil and oil in water.

\subsection{Comparison of slurry indexes before and after solid-liquid separation}

Table 1. The basic properties of waste drilling mud

\begin{tabular}{|c|c|c|c|c|c|c|}
\hline Measure & $\begin{array}{c}\text { Solid } \\
\text { content } / \%\end{array}$ & $\begin{array}{c}\text { Volume } \\
/ \mathrm{mL}\end{array}$ & $\mathrm{PH}$ & $\begin{array}{c}\mathrm{COD} \\
/ \mathrm{mg} \cdot \mathrm{L}^{-1}\end{array}$ & $\begin{array}{c}\text { Total chromium } \\
/ \mathrm{mg} \cdot \mathrm{L}^{-1}\end{array}$ & $\begin{array}{c}\text { Total lead } \\
/ \mathrm{mg} \cdot \mathrm{L}^{-1}\end{array}$ \\
\hline Before & 40.5 & 79.2 & 10.89 & 22564 & 0.0271 & 0.0969 \\
\hline After & 77.5 & 31.5 & 6.72 & 88.7 & 0.0085 & - \\
\hline
\end{tabular}

Table 1 shows that $\mathrm{pH}$, COD and heavy metal content are significantly reduced after solid-liquid separation and meet the national emission requirements. Mud cake volume is more greatly reduced than the mud volume before separation, which can come true the effective realization of solid waste reduction of emissions and saving cost. Because during the slurry solid-liquid separation process, effective the harmful substances in the original slurry enrichment in the separation of the liquid phase, and after the separation of the liquid phase can be further processing and recycling through the film 
processing techniques.

\section{Conclusions}

(i) Mud separation effect by coalescing agent, acidulant and coagulant types and dosage, when the organic gelling agent for A-PA and gel breaker for H20, and dosage respectively for zero point four grams and zero point five grams, drilling mud solid-liquid separation effect best, mud cake of solid content by $40.5 \%$ increased to $77.5 \%$.

(ii) Through the indoor experiment, $\mathrm{pH}, \mathrm{COD}$, heavy metal content reached the national emission requirements, volume decreased by approximately $60 \%$, maximum realized solid waste reduction of emissions, with good economic and social benefits.

\section{References}

1. M. J. Yang, W. L. Liang, Q M Jing, et al. JMP, 23: 109-112,(2003)

2. G. Li, M Zhu, J. L. Qian. IWT, 17: 13-14,17,45,(1997)

3. J. Liu, J. Zhang. EE, 27: 91-93,(2009)

4. J. Q. Chen. SP, 399-402,(2005)

5. G. J. Hu, J. B. Li, G.M. Zeng. JHM, 261C: 470-490,(2013)

6. A. H. Long, Y. X. Sun. WCEE, 15: 165-168,(2003)

7. L. M. Guo,. J. Yuk, X. Chen. EPC, 37:58-61, (2015) 